\title{
Transtornos alimentares e padrão circadiano alimentar: uma revisão
}

\author{
Eating disorders and circadian pattern eating: a review
}

\author{
Fabiana Bernardi ${ }^{1}$, Ana Beatriz Cauduro Harb ${ }^{2}$, Rosa Maria Levandovski ${ }^{3}$, Maria Paz Loayza Hidalgo ${ }^{4}$ \\ ${ }^{1}$ Especialista. Participante, Grupo Cronobiologia Humana, Hospital de Clínicas de Porto Alegre (HCPA), Universidade Federal do Rio Grande do Sul (UFRGS), Porto \\ Alegre, RS. Mestranda, Programa de Ciências Médicas, Faculdade de Medicina, UFRGS. ${ }^{2}$ Mestre. Integrante, Grupo de Cronobiologia Humana, HCPA, UFRGS. Professora, \\ Universidade do Vale do Rio dos Sinos (UNISINOS), São Leopoldo, RS. Doutoranda, Programa de Ciências Médicas, Faculdade de Medicina, UFRGS. ${ }^{3}$ Mestre. Farmacêutica \\ e bioquímica, Hospital Nossa Senhora da Conceição (HNSC), Porto Alegre, RS. Doutoranda, Programa de Ciências Médicas, Faculdade de Medicina, UFRGS. ${ }^{4}$ Doutora. \\ Psiquiatra. Professora adjunta, Departamento de Psiquiatria, Faculdade de Medicina, UFRGS. Serviço de Psiquiatria, HCPA, UFRGS. \\ Trabalho realizado no Hospital de Clínicas Porto Alegre (HCPA), Universidade Federal do Rio Grande do Sul (UFRGS), Porto Alegre, RS.
}

\section{Resumo}

Este artigo tem como objetivo revisar aspectos relacionados a transtornos alimentares e suas relações com as alterações no ritmo circadiano. Realizouse uma busca sistematizada das informações nas bases de dados PubMed usando os seguintes descritores: eating disorders, circadian rhythm, night eating syndrome, binge eating disorder e sleep patterns. Os transtornos alimentares, como a síndrome do comer noturno e o transtorno da compulsão alimentar periódica, têm sido considerados e relacionados a um atraso no ritmo circadiano da ingestão alimentar e saciedade prejudicada. Os ritmos circadianos são aqueles que apresentam um período de $24 \mathrm{~h}$, como, por exemplo, o ciclo sono-vigília, temperatura corporal, atividade e comportamento alimentar. Distúrbios provocados pelas alterações nos horários de sono/vigília influenciam o apetite, a saciedade e, consequentemente, a ingestão alimentar, o que parece favorecer o aumento desses transtornos. Percebe-se que o comportamento alimentar pode ser influenciado por ritmos circadianos. Porém, mais estudos e o maior conhecimento sobre a ritmicidade alimentar podem contribuir com o melhor entendimento do comportamento alimentar atual, atuando na prevenção e/ou tratamento de transtornos alimentares.

Descritores: Transtornos alimentares, ritmo circadiano, síndrome do comer noturno, transtorno da compulsão alimentar periódica, padrões de sono.

\begin{abstract}
This review aims at reviewing aspects related to eating disorders arising from changes in circadian rhythm. There was a systematic search in PubMed databases, using the following descriptors: eating disorders, circadian rhythm, night eating syndrome, binge eating disorder, and sleep patterns. Eating disorders, such as night eating syndrome and binge eating disorder, have been considered and related to a delay in circadian rhythm in food intake and impaired satiety. Circadian rhythms are those that show a period of $24 \mathrm{~h}$, for example, sleep-wake cycle, body temperature, activity and eating behavior. Disorders related to changes in sleep-wake schedules influence the appetite, satiety and consequently food intake, which seems to increase such disorders. Circadian rhythm can influence eating behavior. However, more studies and knowledge about food rhythmicity might contribute to better understanding the current eating behavior, acting in prevention and/or treatment of eating disorders.
\end{abstract}

Keywords: Eating disorders, circadian rhythm, night eating syndrome, binge eating disorder, sleep patterns. 


\section{Introdução}

A etiopatogenia dos transtornos alimentares é complexa. Os fatores biológicos, psicológicos e ambientais, que podem afetar os ritmos circadianos, têm sido considerados no desenvolvimento de transtornos como a síndrome do comer noturno ( $\mathrm{SCN}$ ) e o binge eating disorder (BED), atualmente traduzido para o português como transtorno da compulsão alimentar periódica (TCAP) ${ }^{1}$. A disfunção circadiana hormonal e distúrbios provocados pelas alterações de horários no ciclo sono/vigília influenciam o apetite, a saciedade e, consequentemente, a ingestão alimentar ${ }^{2}$, o que parece favorecer o desenvolvimento desses transtornos.

Os ritmos circadianos, como o ciclo sono/vigília e o comportamento alimentar, são gerenciados por um relógio interno, sincronizado pelo período de $24 \mathrm{~h}$. O sistema circadiano é responsável por manter a sincronização entre comportamentos, sistemas fisiológicos e moleculares, controlando expressões de adipocinas, atividades enzimáticas e hormônios responsáveis pelo controle do processo alimentar. A dessincronização de ritmicidade desse sistema vem sendo estudada nos transtornos alimentares como SCN e TCAP, considerados como exemplos clínicos de dissociação no ritmo circadiano do padrão alimentar e na saciedade ${ }^{3}$.

A SCN tem sido relacionada a um atraso no ritmo circadiano da ingestão alimentar, acompanhada por humor deprimido $^{4,5}$. Foi descrita primeiramente por Stunkard, em um grupo de pacientes obesas que apresentavam características semelhantes, como anorexia matutina, hiperfagia noturna e insônia ${ }^{6}$. Estimativas atuais indicam que 0,5 a 1,5\% da população americana apresenta a SCN. Em clínicas de obesidade, estes índices variam de 6 a 14\%, podendo chegar a $42 \%$ em pacientes que procuram cirurgia para obesidade nos EUA ${ }^{7}$. Pacientes psiquiátricos apresentam uma prevalência de $12 \%$, independentemente do peso $^{8}$. Também tem sido reportada em $15 \%$ de indivíduos que procuram tratamento para TCAP $^{9}$.

O TCAP também foi descrito, primeiramente, na década de 1950, em um grupo de obesos com dificuldade para perder peso e que apresentavam um comportamento alimentar semelhante aos ataques bulímicos dos pacientes com bulimia nervosa ${ }^{10}$. Em 1994, seus critérios diagnósticos foram inclusos no apêndice B do DSM-IV ${ }^{11}$, caracterizando o transtorno pela ingestão de uma grande quantidade de alimentos, seguido pela sensação de perda do controle, tristeza e culpa após comer e ausência de comportamentos compensatórios. A prevalência de TCAP encontra-se em $2 \%$ da população. Em clínicas de obesidade, os índices variam de 9 a $19 \%$ entre pacientes obesos e de 9 a $47 \%$ entre pacientes para cirurgia bariátrica ${ }^{12}$.

A SCN e o TCAP ocorrem mais frequentemente em indivíduos obesos, porém também ocorrem em indivíduos com peso normal, podendo, assim, contribuir para o desenvolvimento e manutenção da obesidade. Têm sido associados com atraso no ritmo circadiano, saciedade prejudicada, humor deprimido, estresse, baixa autoestima e comorbidades psiquiátricas $^{13,14}$.
Levando em conta a influência do ritmo circadiano no comportamento alimentar e desenvolvimento de transtornos alimentares, o objetivo desta revisão foi descrever conceitos e achados relacionados à ritmicidade alimentar, bem como transtornos alimentares decorrentes de alterações no ritmo circadiano.

\section{Método}

Para alcançar o objetivo proposto, realizou-se uma busca sistematizada das informações na base de dados MEDLINE (PubMed) (1955-2009). Para apresentar o tema central deste estudo, buscou-se suporte em estudos experimentais, observacionais e de revisão, usando os seguintes descritores: eating disorders, sleep patterns, circadian rhythm, night eating syndrome, e binge eating disorder. Os descritores foram combinados da seguinte forma: circadian rhythm e night eating syndrome, circadian rhythm e binge eating disorder, night eating syndrome e sleep patterns. A lista de referências bibliográficas dos artigos selecionados também foi utilizada para localização de artigos considerados relevantes. Foram selecionados os artigos cujos fatores em estudo e desfechos estavam relacionados com o escopo desta revisão, limitando para estudos em humanos adultos, escritos em inglês ou espanhol.

\section{Revisão da literatura}

\section{Síndrome do comer noturno}

A SCN é um exemplo clínico de dissociação entre padrões alimentares e sono ${ }^{3}$. Tem sido relacionada a um atraso no ritmo circadiano da ingestão alimentar, acompanhada por humor deprimido ${ }^{4,5}$. A SCN foi descrita primeiramente, em 1955 , em um grupo de pacientes obesas. Stunkard relatou que $64 \%$ destas pacientes mostraram evidências de anorexia matutina, hiperfagia noturna e insônia ${ }^{6}$. Diferentes critérios têm sido usados ao longo dos anos (Harb AB, Levandovski RM, Oliveira C, Hidalgo MP, Night eating syndrome: a correlation study with body mass index and sleep and eating behavior, manuscrito não publicado, 2008). No entanto, o critério mais atual foi estabelecido durante a realização do I Simpósio Internacional sobre Síndrome do Comer Noturno, realizado em abril de $2008^{15}$, o qual definiu o conceito para o novo critério diagnóstico da $\mathrm{SCN}$.

A SCN tem sido associada a mudanças no padrão alimentar e nos fatores neuroendócrinos, que se relacionam com os ritmos circadianos de secreções endócrinas como cortisol, melatonina, adipocinas (leptina e grelina) e distúrbios do sono. Alguns estudos demonstram a diminuição da melatonina durante a noite em indivíduos com $\mathrm{SCN}$ e registram relatos de pacientes com dificuldades para dormir ou então adormecer e manter-se dormindo. Tais respostas levam a considerar que a redução de melatonina possa contribuir para manter a insônia e o humor deprimido $^{16}$. Estudo avaliando comedores noturnos e grupo controle demonstrou que indivíduos com SCN apresentam uma redução 


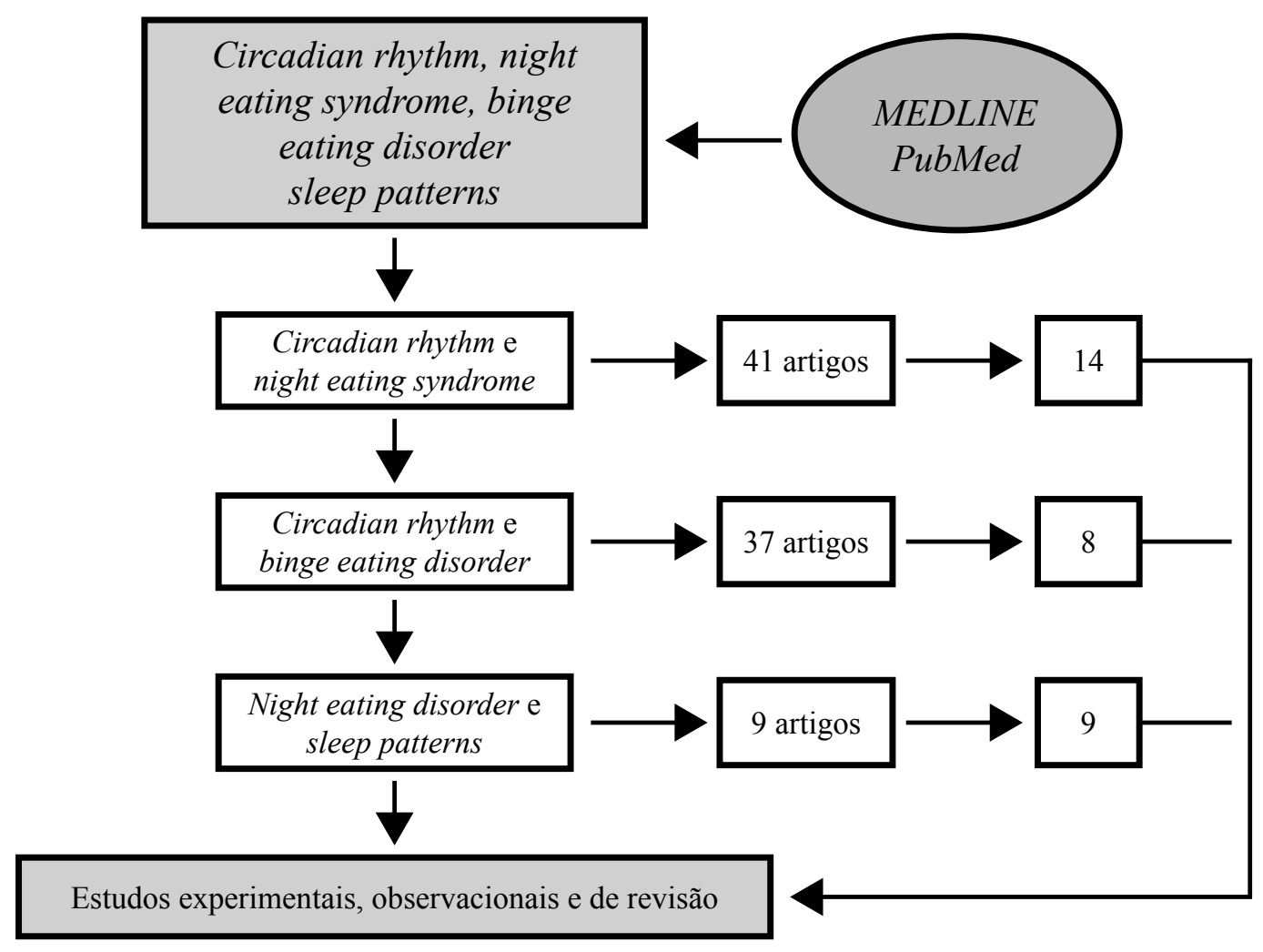

Figura 1 - Fluxograma de identificação dos artigos

na eficiência e qualidade do seu sono e são mais deprimidos do que o grupo controle ${ }^{17}$.

Estudo realizado com indivíduos com SCN (obesos e não obesos) e grupo controle mostrou uma desregulação no ritmo circadiano de melatonina, leptina e cortisol. Os níveis de melatonina e leptina apresentaram-se menores à noite no grupo de comedores noturnos do que no grupo controle, e os níveis de cortisol tendem a ser mais altos durante o dia em pacientes com $\mathrm{SCN}^{16}$. Os níveis de leptina reduzidos à noite podem contribuir para a menor inibição contra os impulsos de fome noturna que interrompem o sono, enquanto o aumento dos níveis de cortisol pode estar relacionado ao estresse presente nesses indivíduos ${ }^{18}$.

A SCN foi descrita inicialmente como uma resposta ao estresse diurno característico de alguns indivíduos obesos e acreditava-se que seus sintomas diminuíam com o alívio do estresse $^{18}$. Os níveis de glicose e insulina parecem estar prejudicados, aumentando o risco para o desenvolvimento de diabetes melito ${ }^{16}$. Estudo realizado com comedores noturnos e grupo controle demonstrou que os níveis de glicose e de insulina elevam-se mais à noite em comedores noturnos; entretanto, estes se encontram diminuídos pela manhã ${ }^{19}$, em resposta ao consumo de uma grande quantidade de carboidratos, uma vez que os comedores noturnos tendem a ingerir principalmente esse nutriente em seus despertares noturnos ${ }^{16,20}$.

Estudo evidenciou que, em indivíduos com hábitos diurnos, a insulina tende aumentar em resposta às refeições, ao passo que, em indivíduos com hábitos noturnos, a insulina tende a estar elevada por um período de tempo maior. Em indivíduos diurnos, os picos de concentração de glicose seguiam o horário das refeições $(9 \mathrm{~h}, 15 \mathrm{~h}$ e $21 \mathrm{~h})$ e os picos de concentração de insulina correspondiam aos de glicose, evidenciando um padrão rítmico de liberação, enquanto em indivíduos com hábitos noturnos os picos ocorriam às $12 \mathrm{~h}$, $21 \mathrm{~h}$ e se estendiam até às $6 \mathrm{~h}$. Ou seja, a concentração de glicose plasmática encontrava-se significativamente mais alta no grupo com estilo de vida noturno ${ }^{21}$. Glicose e insulina possuem ritmos circadianos complementares, mas em comedores noturnos suas fases apresentam-se em atraso, demonstrando dificuldades metabólicas devido à ingestão noturna, principalmente de refeições/lanches ricos em carboidratos ${ }^{22}$.

A grelina tende a estar diminuída à noite em indivíduos com SCN. Os níveis diminuídos de grelina sugerem que a ingestão alimentar suprime seus níveis, ou seja, sua concentração tende a estar diminuída após as refeições, e que níveis mais baixos à noite são uma consequência do aumento do comer noturno ${ }^{19}$. Estudo recente com comedores noturnos e grupo controle, avaliados em laboratório por três noites, apresentou uma fase avançada para o ritmo circadiano da grelina, favorecendo, assim, o aumento do apetite. O estudo sugere que a influência da privação do sono, as alterações no padrão alimentar ou as mudanças no padrão da insulina e/ou glicose podem interferir na desregulação do ritmo da grelina $^{22}$. 
Entre os fatores comportamentais envolvidos na $\mathrm{SCN}$, destacam-se: o horário do consumo de energia; os níveis de humor durante o tempo em que estão acordados; e a frequência de acordar durante a noite ${ }^{23-25}$.

Recentes análises circadianas da ingestão alimentar de indivíduos com SCN demonstraram um significativo atraso, de aproximadamente $1,5 \mathrm{~h}$ na fase de ingestão calórica total e no consumo de nutrientes como carboidratos e gorduras. Esses estudos demonstram que esses indivíduos permanecem comendo por um maior período de tempo durante a noite do que o grupo controle, sofrendo um fenômeno denominado pelos cronobiologistas de arrastamento de fase $\mathrm{e}^{22,26}$. Estudo observacional realizado com indivíduos obesos com SCN e grupo controle demonstrou que os comedores noturnos consomem mais calorias diárias, sendo que $56 \%$ da sua ingestão calórica se dá entre 22 e $6 \mathrm{~h}^{16}$. Porém, há estudos que demonstram que a ingestão calórica total diária é similar à do grupo controle e que a ingestão noturna se mantém maior entre comedores noturnos, demonstrando que estes consomem mais calorias durante o período das 20 às $8 \mathrm{~h}$ e uma tendência a comer poucas calorias das 8 às $20 \mathrm{~h}^{3,19,26}$.

Registra-se também que os comedores noturnos acordam significativamente mais vezes durante a noite do que o grupo controle, sendo que a metade dos despertares é acompanhada da ingestão de alimentos. Além disso, este estudo demonstrou que o humor médio dos pacientes investigados foi mais baixo do que os do grupo controle, registrando-se o dado significativo de que, em $24 \mathrm{~h}$, havia um declínio do humor começando no final da tarde ${ }^{16}$. Inúmeros estudos têm demonstrado que comedores noturnos obesos têm maiores níveis de depressão e baixa autoestima ${ }^{5,7,13,27}$.

A associação entre comedores noturnos e obesidade tem sido muito relatada (Harb AB, Levandovski RM, Oliveira C, Hidalgo MP, Night eating syndrome: a correlation study with body mass index and sleep and eating behavior, manuscrito não publicado, 2008) $)^{28}$. Estudo recente, incluindo 100 participantes, demonstrou associação entre comedores noturnos e índice de massa corporal (IMC) elevado. Esse estudo apontou que, provavelmente, $\mathrm{SCN}$ pode ser um fator de risco para a obesidade e, entre a população obesa, há uma positiva relação entre $\mathrm{SCN}$ e ganho de peso (Harb $\mathrm{AB}$, Levandovski RM, Oliveira C, Hidalgo MP, Night eating syndrome: a correlation study with body mass index and sleep and eating behavior, manuscrito não publicado, 2008).

Estimativas de prevalência sugerem que a SCN é mais comum entre indivíduos obesos, porém nem todos os indivíduos com SCN apresentam excesso de peso ${ }^{20,29,30}$. Lundgren et al. ${ }^{30}$, em estudo descritivo com comedores noturnos não obesos e grupo controle, evidenciaram que comedores noturnos não obesos também têm implicações negativas na saúde, sendo associado com comportamentos alimentares, humor, estresse e distúrbios do sono. Não há diferenças entre comedores noturnos obesos e não obesos para apetite matutino, hiperfagia vespertina, dificuldades para dormir e humor. A única diferença é que comedores noturnos não obesos são significativamente mais jovens e relatam maior dificuldade para manter o sono, ou seja, acordam mais vezes e ingerem mais alimentos à noite. Os autores também sugerem a necessidade de estudo longitudinal, com maior número de participantes para determinar se o comer noturno determina o ganho de peso durante longos períodos.

\section{Transtorno da compulsão alimentar periódica}

Padrões irregulares do comportamento alimentar também têm sido observados em indivíduos com TCAP, em alguns estudos sendo relacionado ao sobrepeso e obesidade, o que exerce importante papel na saúde e peso corporal.

TCAP é caracterizado pelo consumo de uma grande quantidade de alimentos, em até $2 \mathrm{~h}$, seguido pela sensação de perda do controle, tristeza e culpa após comer e ausência de comportamentos compensatórios ${ }^{31,32}$. Em 1994, foi incluído no apêndice B do DSM-IV ${ }^{11}$, sob a forma de transtorno que necessita de maiores estudos para melhor caracterização. Inclui anormalidades fisiológicas na ação de hormônios que influenciam a ingestão alimentar, como insulina, leptina, grelina, entre outros ${ }^{31,33}$.

Tem sido observado, na prática clínica, que os pacientes obesos relatam o estresse como um fator para o início da compulsão alimentar e outras desordens alimentares. Entretanto, o mecanismo para essa relação ainda não está esclarecido ${ }^{31,35}$. Estudo avaliando mulheres obesas com TCAP e sem TCAP demonstrou que o cortisol basal matutino foi significativamente maior nos indivíduos com TCAP ${ }^{19}$. Gluck ${ }^{31}$, em revisão, avaliou que muitos estudos relacionam estresse, cortisol, ingestão alimentar e gordura abdominal. Outro estudo, mais recente, avaliando níveis noturnos de cortisol salivar em obesas com e sem TCAP, demonstrou que a presença de TCAP não foi associada com o aumento dos níveis da secreção de cortisol. A limitação desse estudo pode estar na escolha, classificação dos indivíduos e tamanho da amostra, limitando o poder do estudo em demonstrar a diferença (erro $\beta)^{32}$.

Estresse e emoções negativas têm sido associados com irregularidades na ingestão alimentar em humanos. Sabe-se que peptídeos como a leptina e a grelina, entre outros, podem estar envolvidos na ingestão alimentar e na regulação da resposta ao estresse. A interação entre grelina e cortisol tem sido estudada em humanos. Estudo avaliando obesos e não obesos com TCAP e sem TCAP indicou que o estresse pode induzir um aumento nos níveis de grelina plasmática, associado a níveis séricos de cortisol ${ }^{23}$. Porém, a maioria dos estudos demonstram níveis de grelina significativamente menores em jejum e com um pequeno declínio após as refeições em indivíduos com TCAP $24,35-37$.

Alguns estudos têm relatado aumento da concentração de leptina em pacientes obesos com TCAP, sugerindo que a leptina pode ser um sensível marcador metabólico das mudanças ocorridas na compulsão alimentar e que o aumento dos níveis dessa adipocina pode estar envolvido no desenvolvimento e/ou manutenção do comportamento compulsivo ${ }^{24,25}$. Estudo com 30 mulheres obesas (TCAP e não TCAP), com o objetivo 
de avaliar concentrações de leptina e correlacionar dados e atitudes alimentares, demonstrou relação positiva entre aumento dos níveis desta com gordura corporal e padrões alimentares, influenciando a frequência de compulsões alimentares, aumento de energia ingerida e tecido adiposo ${ }^{25}$.

Os níveis de glicose e insulina na TCAP são os mesmos que em indivíduos obesos ${ }^{24}$, e elevados quando comparados a indivíduos com peso normal ${ }^{33,38}$.

TCAP ocorre em indivíduos eutróficos, mas mais frequentemente em indivíduos com sobrepeso ou obesidade e pode contribuir para o desenvolvimento e/ou manutenção da obesidade $^{39}$. Estudos também têm demonstrado que comedores noturnos podem ser compulsivos. Revisão demonstrou que comedores noturnos com um subtipo para TCAP e o duplo diagnóstico têm sido associados com IMC elevado ${ }^{5}$. Outro estudo comparando indivíduos com TCAP, comedores noturnos e grupo controle demonstrou que TCAP e SCN relatam mais atitudes e comportamentos inadequados para o padrão alimentar e são mais deprimidos do que o grupo controle ${ }^{12}$.

Há poucos estudos controlados que avaliem TCAP e SCN. Estudo encontrou em pacientes com TCAP e SCN maiores níveis de restrição dietética e problemas alimentares do que quando comparados a um grupo controle. TCAP tem sido relacionado a altos níveis de restrição alimentar, seguidos por momentos de compulsão alimentar, demonstrando um padrão alimentar irregular ${ }^{12,13,31,40}$. Napolitano et al. ${ }^{13}$ compararam características psicológicas e comportamentais de indivíduos com TCAP, comedores noturnos, indivíduos com diagnóstico para TCAP e SCN e indivíduos sem transtorno alimentar, demonstrando que comedores noturnos apresentam menor desinibição quando comparados a indivíduos com TCAP, além de que pacientes com ambas desordens relatam maior ansiedade. $\mathrm{O}$ estudo sugere que as duas desordens podem estar associadas a comorbidades psiquiátricas e reforça a necessidade de futuros estudos.

Percebe-se que as mudanças na ingestão alimentar podem induzir atraso na regulação metabólica ${ }^{22}$ e que os ritmos circadianos do ciclo sono/vigília, hormônios, apetite e estresse podem estar relacionados a transtornos alimentares e alterar os níveis e o padrão de liberação ao longo do dia de componentes responsáveis pelo controle do processo alimentar e, consequentemente, promover alterações no padrão alimentar.

\section{Ritmo circadiano alimentar}

A ritmicidade alimentar circadiana é herdada, espécieespecífica, uma vez que o homem é basicamente diurno. Está sincronizado ao ciclo claro/escuro ${ }^{2,41,42}$ e aos níveis de cortisol, serotonina, leptina, citocinas, entre outros ${ }^{2,41}$. Além disso, o ritmo social imprime marcado efeito na regulação da alimentação, pois a sociedade contemporânea funciona $24 \mathrm{~h}$ e seu impacto na quantidade, qualidade e horários da alimentação tem sido inexorável. Como exemplo, cita-se o atraso da alimentação para adaptação aos horários de trabalho ${ }^{41}$.

Os ritmos circadianos são aqueles que apresentam um período de $24 \mathrm{~h}$, como, por exemplo, o ciclo sono-vigília e a temperatura corporal. A sincronização desses ritmos é realizada pelo núcleo supraquiasmático, localizado no hipotálamo (sistema nervoso central), responsável pela coordenação dos ritmos de comportamento e fisiologia ${ }^{3}$. Existem algumas situações em que ocorre a dessincronização da ritmicidade, tendo em vista que a quantidade, a qualidade e o horário das refeições parecem ser determinados por reflexos condicionados, pela rotina e pela sensação de que "é hora de comer". As refeições atuariam como um Zeitgeber, neologismo alemão que significa "marcador de tempo", indicando que as refeições seriam determinadas por uma organização social ou sincronização do ritmo biológico. Para a maioria dos organismos, o Zeitgeber mais importante dos ritmos circadianos é o ciclo claro/escuro. No entanto, outros fatores cíclicos também agem como sincronizadores, como, por exemplo, os ciclos sociais, de temperatura e alimentação. Os Zeitgeber agem interferindo nas funções digestivas e de absorção e nas variações da concentração de carboidratos, aminoácidos e lipídeos na corrente sanguínea ao longo do diaª .

Ritmos circadianos e diurnos afetam a ingestão alimentar. Muitos comportamentos, incluindo a ingestão energética, variam em nível e intensidade ao longo do dia nesses processos. Estudos recentes têm mostrado que a quantidade calórica das refeições, os intervalos pós-refeições e os níveis de saciedade diminuem durante o dia. $\mathrm{O}$ horário das refeições pode ser relacionado com a ingestão total durante o dia. Os resultados desses estudos sugerem que ingerir um valor calórico maior pela manhã (ou primeira parte do dia) pode resultar em uma diminuição da ingestão total durante o dia, enquanto a ingestão de uma grande quantidade energética à noite pode resultar em um aumento do total da ingestão ${ }^{43}$.

A alteração dos níveis de adipocinas é considerada um importante mecanismo capaz de alterar o padrão da ingestão alimentar e levar a desajustes nutricionais. A ritmicidade e o sincronismo na secreção destas são importantes para o padrão diário das refeições. Estudos indicam que um padrão rítmico recíproco entre a leptina e a grelina estabelece ritmicidade no sistema neuropeptídeo Y (NPY), que é o caminho final comum para a expressão do apetite no hipotálamo. Assim, a leptina inibe tanto a secreção de grelina quanto o estímulo de alimentação por esta, indicando que a leptina desempenha o papel de comunicação de realimentação entre a periferia e o hipotálamo para a homeostase da massa corporal ${ }^{44}$.

A leptina é secretada pelo tecido adiposo. A ação desta no sistema nervoso central promove a redução da ingestão alimentar e o aumento do gasto energético, além de regular a função neuroendócrina e o metabolismo da glicose e de gorduras. A expressão da leptina é controlada por diversas substâncias, como insulina, glicorticoides e citocinas pró-inflamatórias. Assim, um aumento nos níveis desta reduz a ingestão alimentar, enquanto baixos níveis induzem a alimentação ${ }^{16,18}$. Os níveis de concentração da leptina encontram-se aumentados durante a noite em indivíduos com hábitos diurnos e peso saudável ${ }^{21}$.

A concentração de insulina, hormônio secretado pelo pâncreas, é estimulada pela glicose plasmática após as refeições. 
Estudo demonstrou, em indivíduos de hábitos diurnos, que os picos de concentrações de glicose ocorriam às 9 , às 15 e às $21 \mathrm{~h}$, seguindo o ritmo normal das refeições (café-damanhã, almoço e janta), enquanto em indivíduos de hábitos noturnos, as concentrações de glicose aumentaram à noite, a partir das $21 \mathrm{~h}$ até as $6 \mathrm{~h}^{21}$.

A grelina é sintetizada, primeiramente, pelo estômago e responsável pelo aumento da secreção do hormônio do crescimento (growth hormone, GH). Age também no controle do gasto energético, controle da secreção ácida e da motilidade gástrica e influencia a função endócrina pancreática e o metabolismo da glicose. Está envolvida na sinalização dos centros hipotalâmicos que regulam a ingestão alimentar e o balanço energético. Níveis circulantes de grelina encontram-se aumentados durante o jejum prolongado e em estado de hipoglicemia e têm sua concentração diminuída após a refeição.

Os nutrientes presentes na refeição, e não a sua quantidade, são os responsáveis pelo aumento ou decréscimo pós-prandial. Os níveis de grelina diminuem na presença de carboidratos e aumentam com a ingestão de proteínas e lipídeos. Esses níveis são influenciados pelas mudanças no balanço energético e tem sido mostrado que a insulina pode ter um importante papel na diminuição da grelina após as refeições ${ }^{45,46}$.

O cortisol é um hormônio corticosteroide produzido pela glândula suprarrenal que está envolvido na resposta ao estresse e pode ter um papel importante no comportamento alimentar, além de ser associado com aumento da ingestão energética em indivíduos saudáveis ${ }^{47}$. A produção de cortisol tem um ritmo circadiano que depende da estimulação do hormônio adrenocorticotrópico (adrenocorticotropic hormone, $\mathrm{ACTH}$ ). Seu nível encontra-se elevado próximo ao início das atividades diárias, decaindo ao longo das $24 \mathrm{~h}$.

\section{Ritmo sono-vigilia e comportamento alimentar}

O ciclo sono/vigília é um dos ritmos mais estudados; a regulação da maioria dos comportamentos e atividades fisiológicas depende do adormecer ou acordar ${ }^{47}$. O principal hormônio envolvido no ciclo sono/vigília é a melatonina, e o adormecer é um sinal de início da secreção, ou seja, tem seu início de elevação ao anoitecer e queda no fim da noite. As condições de sono podem influenciar a secreção de melatonina. Estudo realizado em indivíduos com hábitos noturnos demonstrou uma diminuição na produção de melatonina ${ }^{21}$. A diminuição dos níveis de melatonina tem sido associada com o sono prejudicado, depressão e $\mathrm{SCN}^{16}$.

A privação parcial do sono parece exercer influência nos níveis de vários hormônios. Estudos têm mostrado que a restrição do sono por alguns dias é o suficiente para haver o aumento nos níveis de glicose e diminuição da ação da insulina, mostrando que longos períodos de sono prejudicado pode ser um fator de risco para o desenvolvimento do diabetes melito. A privação do sono também resultou em níveis menores de leptina, aumento dos níveis de grelina, cortisol e do apetite ${ }^{47}$. Em situações normais de sono, os níveis de leptina e grelina encontram-se aumentados durante o sono ${ }^{44}$, enquanto os níveis de cortisol tendem a estar menores à noite, elevando-se no início da manhã.

$\mathrm{O}$ aumento do apetite e a preferência por alimentos mais calóricos parecem estar presentes na privação do sono. O padrão alimentar noturno rico em carboidratos aumenta o transporte de triptofano para o cérebro, convertendo em serotonina e resultando em uma maior facilidade para dormir ${ }^{16}$. Estudo mostrou que o apetite por nutrientes que continham alta quantidade de carboidratos, incluindo doces, biscoitos salgados e tubérculos, aumentou de 33 para $45 \%$, mas o apetite por frutas, vegetais e alimentos com alta quantidade de proteínas foi pouco afetado ${ }^{44}$. Lennernas et al..$^{48}$ observaram uma grande preferência pelo consumo de lanches rápidos e calóricos durante o horário de trabalho nos trabalhadores noturnos.

\section{Conclusão}

A revisão realizada apresenta a influência dos ritmos circadianos e a importância de vários hormônios regulatórios no comportamento alimentar e desenvolvimento de transtornos alimentares. Alterações comportamentais e neuroendócrinas estão presentes nos transtornos alimentares, e um atraso no ritmo circadiano da ingestão alimentar e saciedade prejudicada podem levar ao desenvolvimento de transtornos alimentares, como SCN e TCAP, sendo associados, em muitos estudos, com IMC elevado, humor deprimido, estresse e ansiedade.

A ritmicidade e o sincronismo na secreção dos níveis de hormônios regulatórios são importantes para o padrão diário das refeições, alterando o padrão da ingestão alimentar e resultando em desajustes nutricionais. Estudos indicam que um padrão rítmico recíproco entre a leptina e a grelina estabelece ritmicidade para a expressão do apetite. Assim, a leptina desempenha um papel de comunicação de realimentação entre a periferia e o hipotálamo para a homeostase da massa corporal. Assim como as alterações de sono, também resultou em níveis menores de leptina, aumento dos níveis de grelina, cortisol e aumento do apetite e a preferência por alimentos mais calóricos. A diminuição na produção de melatonina, associada com a restrição do sono e o aumento nos níveis de glicose e diminuição da ação da insulina, também foi observada nos estudos, resultando na dessincronização da ritmicidade alimentar. As mudanças na ingestão alimentar podem induzir atraso na regulação metabólica dos ritmos circadianos e foram relacionadas nos estudos a transtornos alimentares como a SCN e TCAP, que podem contribuir para o desenvolvimento e manutenção da obesidade.

Porém, os estudos atuais não definem se fatores como IMC, estresse e humor são causa ou consequência da desordem alimentar. Estudos prospectivos, com maior número de participantes, controle de fatores confundidores, uso de instrumentos validados, consensos para critérios diagnósti$\cos$, inclusão da $\mathrm{SCN}$ no $\mathrm{DSM}^{49}$, e utilização de questionários adequados, como o Night Eating Questionnaire (NEQ) ${ }^{50}$ 
e Binge Eating Scale (BES) $)^{51}$, traduzidos e validados para a população brasileira, ainda são necessários para elucidar essa relação. Assim, é necessário que sejam realizadas novas pesquisas para elucidar o estudo dos ritmos circadianos no comportamento alimentar e aprofundar o conhecimento nessa área, contribuindo com o melhor entendimento do comportamento alimentar atual, agindo na prevenção e/ou tratamento de transtornos alimentares.

\section{Referências}

1. Lo Sauro C, Ravaldi C, Cabras PL, Faravelli C, Ricca V. Stress, hypothalamic-pituitary-adrenal axis and eating disorders. Neuropsychobiology. 2008;57(3):95-115.

2. Hidalgo MS, Zanette C, Nunes P, Voltolini S, Pedrotti M, et al. Revisão sobre cronobiologia e aspectos cognitivo-comportamentais relacionados à cronotipos. Rev Psiquiatr RS. 2003;25:174-81.

3. O'Reardon JP, Ringel BL, Dinges DF, Allison KC, Rogers NL, Martino NS, et al. Circadian eating and sleeping patterns in the night eating syndrome. Obes Res. 2004;12(11):1789-96.

4. Stunkard A, Allison K, Lundgren J. Issues for DSM-V: night eating syndrome. Am J Psychiatry. 2008;165(4):424

5. Vinai P, Allison KC, Cardetti S, Carpegna G, Ferrato N, Masante D, et al. Psychopathology and treatment of night eating syndrome: a review. Eat Weight Disord. 2008;13(2):54-63.

6. Stunkard AJ, Grace WJ, Wolff HG. The night-eating syndrome; a pattern of food intake among certain obese patients. Am J Med. 1955;19(1):78-86.

7. Howell MJ, Schenck CH, Crow SJ. A review of nighttime eating disorders. Sleep Med Rev. 2009;13(1):23-34.

8. Lundgren JD, Allison KC, Crow S, O'Reardon JP, Berg KC, Galbraith J, et al. Prevalence of the night eating syndrome in a psychiatric population. Am J Psychiatry. 2006;163(1):156-8.

9. O'Reardon JP, Peshek A, Allison KC. Night eating syndrome: diagnosis, epidemiology and management. CNS Drugs. 2005;19(12):997-1008.

10. Stunkard AJ. Eating patterns and obesity. Psychiatr Q. 1959;33:284-95.

11. American Psychiatric Association. Diagnostic and statistical manual of mental disorders. 4th ed. Washington (DC): APA; 1994.

12. Allison KC, Grilo CM, Masheb RM, Stunkard AJ. Binge eating disorder and night eating syndrome: a comparative study of disordered eating. J Consult Clin Psychol. 2005;73(6):1107-15.

13. Napolitano MA, Head S, Babyak MA, Blumenthal JA. Binge eating disorder and night eating syndrome: psychological and behavioral characteristics. Int $\mathrm{J}$ Eat Disord. 2001;30(2):193-203

14. Tanofsky-Kraff M, Yanovski SZ. Eating disorder or disordered eating? Non-normative eating patterns in obese individuals. Obes Res. 2004;12(9):1361-6.

15. Allison KC, Lundgren JD, O'Reardon JP, Geliebter A, Gluck ME, Vinai P, et al. Proposed diagnostic criteria for night eating syndrome. Int J Eat Disord. 2009 Apr 17. [Epub ahead of print]

16. Birketvedt GS, Florholmen J, Sundsfjord J, Osterud B, Dinges D, Bilker W, et al. Behavioral and neuroendocrine characteristics of the night-eating syndrome. JAMA. 1999;282(7):657-63.

17. Rogers NL, Dinges DF, Allison KC, Maislin G, Martino N, O'Reardon JP, et al. Assessment of sleep in women with night eating syndrome. Sleep. 2006;29(6):814-9.

18. Stunkard AJ, Allison KC. Two forms of disordered eating in obesity: binge eating and night eating. Int J Obes Relat Metab Disord. 2003;27(1):1-12.

19. Allison KC, Ahima RS, O'Reardon JP, Dinges DF, Sharma V, Cummings DE, et al. Neuroendocrine profiles associated with energy intake, sleep, and stress in the night eating syndrome. J Clin Endocrinol Metab. 2005;90(11):6214-7.

20. Striegel-Moore RH, Franko DL, Thompson D, Affenito S, May A, Kraemer HC. Exploring the typology of night eating syndrome. Int J Eat Disord. 2008;41(5):411-8.

21. Qin LQ, Li J, Wang Y, Wang J, Xu JY, Kaneko T. The effects of nocturnal life on endocrine circadian patterns in healthy adults. Life Sci. 2003;73(19):2467-75.

22. Goel N, Stunkard AJ, Rogers NL, Van Dongen HP, Allison KC, O'Reardon JP, et al. Circadian rhythm profiles in women with night eating syndrome. J Biol Rhythms. 2009;24(1):85-94.

23. Rouach V, Bloch M, Rosenberg N, Gilad S, Limor R, Stern N, et al. The acute ghrelin response to a psychological stress challenge does not predict the poststress urge to eat. Psychoneuroendocrinology. 2007;32(6):693-702.
24. Geliebter A, Gluck ME, Hashim SA. Plasma ghrelin concentrations are lower in binge-eating disorder. J Nutr. 2005;135(5):1326-30.

25. Adami GF, Campostano A, Cella F, Scopinaro N. Serum leptin concentration in obese patients with binge eating disorder. Int J Obes Relat Metab Disord. 2002;26(8):1125-8

26. Boston RC, Moate PJ, Allison KC, Lundgren JD, Stunkard AJ. Modeling circadian rhythms of food intake by means of parametric deconvolution: results from studies of the night eating syndrome. Am J Clin Nutr. 2008;87(6):1672-7.

27. Gluck ME, Geliebter A, Satov T. Night eating syndrome is associated with depression, low self-esteem, reduced daytime hunger, and less weight loss in obese outpatients. Obes Res. 2001;9(4):264-7.

28. Colles SL, Dixon JB, O'Brien PE. Night eating syndrome and nocturnal snacking: association with obesity, binge eating and psychological distress. Int J Obes (Lond). 2007;31(11):1722-30.

29. Marshall HM, Allison KC, O'Reardon JP, Birketvedt G, Stunkard AJ. Night eating syndrome among nonobese persons. Int J Eat Disord. 2004;35(2):217-22.

30. Lundgren JD, Allison KC, O'Reardon JP, Stunkard AJ. A descriptive study of non-obese persons with night eating syndrome and a weight-matched comparison group. Eat Behav. 2008;9(3):343-51.

31. Gluck ME. Stress response and binge eating disorder. Appetite 2006;46(1):26-30.

32. Coutinho WF, Moreira RO, Spagnol C, Appolinario JC. Does binge eating disorder alter cortisol secretion in obese women? Eat Behav. 2007;8(1):59-64.

33. Taylor AE, Hubbard J, Anderson EJ. Impact of binge eating on metabolic and leptin dynamics in normal young women. J Clin Endocrinol Metab. 1999;84(2):428-34.

34. Gluck ME, Geliebter A, Hung J, Yahav E. Cortisol, hunger, and desire to binge eat following a cold stress test in obese women with binge eating disorder. Psychosom Med. 2004;66(6):876-81.

35. Geliebter A, Hashim SA, Gluck ME. Appetite-related gut peptides, ghrelin, PYY, and GLP-1 in obese women with and without binge eating disorder (BED). Physiol Behav. 2008;94(5):696-9.

36. Monteleone P, Fabrazzo M, Tortorella A, Martiadis V, Serritella C, Maj M. Circulating ghrelin is decreased in non-obese and obese women with binge eating disorder as well as in obese non-binge eating women, but not in patients with bulimia nervosa. Psychoneuroendocrinology. 2005;30(3):243-50.

37. Geliebter A, Yahav EK, Gluck ME, Hashim SA. Gastric capacity, test meal intake, and appetitive hormones in binge eating disorder. Physiol Behav. 2004;81(5):735-40.

38. Monteleone P, Martiadis V, Fabrazzo M, Serritella C, Maj M. Ghrelin and leptin responses to food ingestion in bulimia nervosa: implications for binge-eating and compensatory behaviours. Psychol Med. 2003;33(3):1387-94.

39. Allison KC, Crow SJ, Reeves RR, West DS, Foreyt JP, Dilillo VG, et al. Binge eating disorder and night eating syndrome in adults with type 2 diabetes. Obesity (Silver Spring). 2007;15(5):1287-93.

40. Adami GF, Campostano A, Marinari GM, Ravera G, Scopinaro N. Night eating in obesity: a descriptive study. Nutrition. 2002;18(7-8):587-9.

41. Waterhouse J, Buckley P, Edwards B, Reilly T. Measurement of, and some reasons for, differences in eating habits between night and day workers. Chronobiol Int. 2003;20(6):1075-92.

42. Harthoorn LF, Sañé A, Nethe M, Van Heerikhuize JJ. Multi-transcriptional profiling of melanin-concentrating hormone and orexin-containing neurons. Cell Mol Neurobiol. 2005;25(8):1209-23.

43. de Castro JM. The time of day of food intake influences overall intake in humans. J Nutr. 2004;134(1):104-11.

44. Crispim CA, Zalcman I, Dáttilo M, Padilha HG, Tufik S, de Mello MT. Relação entre sono e obesidade: uma revisão da literatura. Arq Bras Endocrinol Metab. 2007;51(7):1041-9

45. Romero CE, Zanesco A. O papel dos hormônios leptina e grelina na gênese da obesidade. Rev Nutr. 2006;19(1):85-91.

46. Yildiz BO, Suchard MA, Wong ML, McCann SM, Licinio J. Alterations in the dynamics of circulating ghrelin, adiponectin, and leptin in human obesity. Proc Natl Acad Sci U S A. 2004;101(28):10434-39.

47. Laposky AD, Bass J, Kohsaka A, Turek FW. Sleep and circadian rhythms: key components in the regulation of energy metabolism. FEBS Lett. 2008;582(1):142-51.

48. Lennernas MA, Akerstedt T, Hagman U, Bruce A, Hambraeus L. A new approach for evaluation of meal quality and meal patterns. J Human Nutr Diet. 1993;6(3):261-73.

49. Striegel-Moore RH, Franko DL, May A, Ach E, Thompson D, Hook JM. Should night eating syndrome be included in the DSM? Int J Eat Disord. 2006;39(7):544-9.

50. Harb AB, Caumo W, Hidalgo MP. [Translation and adaptation of the Brazilian version of the Night Eating Questionnaire]. Cad Saude Publica 2008;24(6):1368-76.

51. Freitas S, Lopes CS, Coutinho W, Appolinario JC. Tradução e adaptação para o português da Escala de Compulsão Alimentar Periódica. Rev Bras Psiquiatr. 2001;23(4):215-20. 